\title{
Manipulation of Rat Brain Fatty Acid Composition Alters Volatile Anesthetic Potency
}

\author{
Alex S. Evers, William J. Elliott, James B. Lefkowith, and Phillp Needleman \\ Departments of Anesthesiology and Pharmacology, Washington University School of Medicine, St. Louis, Missouri 63110
}

\begin{abstract}
The molecular mechanism of volatile anesthetic action remains unknown. Attempts to elucidate this mechanism have been complicated by the absence of models in which changes in neuronal cellular properties can be correlated with changes in whole animal anesthetic effect. In this study we describe a model where dietinduced alterations in rat brain fatty acid composition are correlated with alterations in volatile anesthetic potency. Rats maintained on a fat-free diet showed significant depletion of ar-

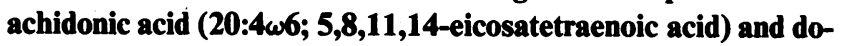
cosahexaenoic acid (22:6 $\omega 3 ; 4,7,10,13,16,19$,-docosahexaenoic acid) in brain, and a corresponding increase in Mead acid (20: $3 \omega 9 ; 5,8,11$-eicosatrienoic acid). These fat-deprived rats were significantly more sensitive to all volatile anesthetics tested than were age-controlled rats on a normal diet. Parenteral supplementation of the fat-deprived animals with linolenic acid (18: $3 \omega 3,9,12,15$-octadecatrienoic acid) completely reconstituted the docosahexaenoic acid content of brain without affecting anesthetic sensitivity. In contrast, supplementation of the fat-deprived rats with linoleic acid (18:2 $\omega 6 ; 9,12$-octadecadienoic acid) caused a dramatic decrease in anesthetic sensitivity, but only a small change in whole brain arachidonate content. Further analysis revealed that linoleate supplementation of fat-deprived animals resulted in a preferential normalization of the arachidonate content of brain phosphatidylinositol as compared with other brain phosphoglycerides. These results demonstrate for the first time a correlation between changes in membrane composition and anesthetic effect, and indicate that the precise fatty acid composition (perhaps in specific phospholipids) of brain is important in the mechanism of volatile anesthetic action.
\end{abstract}

\section{Introduction}

Despite almost a century of research, the molecular mechanism of volatile anesthetic action remains unknown. The experimental observation that the potency of an anesthetic correlates with its partition coefficient between olive oil and water has led to the working hypothesis that all general anesthetics cause a common structural alteration in excitable membranes (1). One school of thought suggests that all anesthetics interact with neuronal membrane lipids, resulting in a common biophysical alteration

Address reprint requests to Dr. Evers.

Received for publication 27 August 1985.

J. Clin. Invest.

(c) The American Society for Clinical Investigation, Inc.

0021-9738/86/03/1028/06 \$1.00

Volume 77, March 1986, 1028-1033 of the lipid bilayer (2). Such alterations in the physical state of the bilayer are theorized either to directly alter membrane function, or to secondarily alter membrane protein structure and function $(3,4)$. The lack of correlation between anesthetic effect and measurable perturbations of model lipid bilayer structures has led others to hypothesize that anesthetics exert their effect directly on a neuronal membrane protein(s), resulting in altered neuronal function $(5,6)$.

Designing experiments to distinguish between the various theories of anesthetic mechanism is complicated by the fact that the anesthetic effect is a whole animal phenomenon. While anesthetics cause a variety of physical perturbations in model membrane systems, and a multitude of biochemical changes in in vitro systems, it is unclear whether these effects are directly related to in vivo anesthetic action (7). In an attempt to gain greater insight into molecular mechanisms of anesthetic action, we have utilized a dietary approach to alter the fatty acid composition of rat brain phospholipids and determined the effects of these manipulations on volatile anesthetic potency in intact animals.

The rationale for our fatty acid manipulation is based on the fact that vertebrates synthesize several non-interconvertible families of polyunsaturated fatty acids by chain elongation/desaturation of specific precursor fatty acids (8). The three major families of polyunsaturated fatty acids are the linolenic $(\omega 3)$, linoleic $(\omega 6)$, and oleic $(\omega 9)$ families having terminal double bonds at three, six, or nine carbons, respectively, from the methyl $(\omega)$ carbon of the fatty acid. In vertebrates, the $\omega 3$ and $\omega 6$ series of polyunsaturated fatty acids (essential fatty acids) are dietary requirements and in their absence the body synthesizes excess $\omega 9$ series fatty acids (8). By placing weanling rats on a fat-free diet, we effect replacement of $\omega 3$ and $\omega 6$ series fatty acids in the brain with $\omega 9$ series fatty acids. Subsequent parenteral supplementation of these fat-deprived animals with either linoleic acid $(\omega 6)$ or linolenic acid $(\omega 3)$ allows us to study animals with specific deficiencies of either $\omega 3$ or $\omega 6$ series polyunsaturated fatty acids in brain.

\section{Methods}

Materials. Polyunsaturated fatty acid methyl esters (PUFA mix no. 1) for gas chromatography standards were purchased from Supelco, Inc: (Houston, TX). Heneicosanoic acid (21:0) and its methyl ester, methyl palmitoleate, phospholipid standards, methyl linolenate, ethyl linoleate, and methanolic $\mathrm{BF}_{3}$ were purchased from Sigma Chemical Co. (St. Louis, MO). Silica gel plates for isolation of fatty acid methyl esters $(20 \times 20$ $\mathrm{cm}, 0.25 \mathrm{~mm}$ ) were purchased from Analabs (North Haven, CT). Silica gel plates for phospholipid separation $(10 \times 20 \mathrm{~cm}$, high performance grade) were purchased from MCB reagents (Gibbstown, NJ).

Diets used in the study were purchased from Purina Test Diets 
Table I.

A. Anesthetic Potencies and Induction Times in EFAD Rats and Age-matched Rats on a Normal Diet (Normal)

\begin{tabular}{|c|c|c|c|c|c|c|}
\hline & \multirow{2}{*}{$\begin{array}{l}\text { Relative lipid solubility } \\
\text { (olive oil/gas partition } \\
\text { coefficient) (15) }\end{array}$} & \multicolumn{2}{|l|}{ ED so values } & \multirow{2}{*}{$\begin{array}{l}\text { Percent change } \\
\text { of } E D_{90}\end{array}$} & \multicolumn{2}{|c|}{ Induction time } \\
\hline & & Normal & EFAD & & Normal & EFAD \\
\hline & & vol \% & vol\% & & $s$ & $s$ \\
\hline Methoxyflurane $(n=9)$ & 970 & $0.46 \pm 0.02$ & $0.31 \pm 0.02 \ddagger$ & $-33 \%$ & $600 \pm 116$ & $520 \pm 52$ \\
\hline Halothane $(n=9)$ & 224 & $1.24 \pm 0.04$ & $1.0 \pm 0.06 \ddagger$ & $-19 \%$ & $118 \pm 6$ & $134 \pm 15$ \\
\hline Isoflurane $(n=9)$ & 98 & $1.62 \pm 0.01$ & $1.34 \pm 0.07 \ddagger$ & $-17 \%$ & $74 \pm 7$ & $76 \pm 17$ \\
\hline Cyclopropane $(n=9)$ & 12 & $17.3 \pm 0.4$ & $15.6 \pm 0.5 \ddagger$ & $-10 \%$ & $88 \pm 11$ & $92 \pm 18$ \\
\hline
\end{tabular}

B. Halothane Potency Measured in a Second Group of EFAD Rats Compared with Potency in Weight-matched Rats on a Normal Diet (Normal)

\begin{tabular}{lllll}
\hline & & & & \\
\cline { 2 - 5 } & Normal diet & EFAD diet & $\begin{array}{l}\text { EFAD diet + linolenate } \\
(18: 3 \omega 3)\end{array}$ & $\begin{array}{l}\text { EFAD diet }+ \text { linoleate } \\
(18: 2 \omega 6)\end{array}$ \\
\hline Halothane $(n=10)$ & vol \% & vol \% & vol \% & vol \% \\
& $1.21 \pm 0.02$ & $0.94 \pm 0.02 \S$ & $0.92 \pm 0.03 \S$ & $1.41 \pm 0.04 \ddagger$
\end{tabular}

(A) Anesthetic potency was measured by exposing rats to incrementally reduced concentrations of anesthetic gas and determining the concentration at which the rats responded to a noxious stimulus. $\mathrm{ED}_{\mathbf{s 0}}$ values are expressed as volumes percent anesthetic gas (in 100\% oxygen) \pm SEM. Induction times were determined by exposing rats to a fixed concentration of anesthetic gas and measuring the time until the rats ceased to flick their tails in response to a tail clamp. Values are expressed as the mean \pm SEM. $(B)$ Halothane potency was redetermined in the EFAD animals following parenteral supplementation first with linolenic acid (EFAD $+18: 3 \omega 3)$ and subsequently with linoleic acid $(18: 2 \omega 6) .^{*} P<0.02$; $\ddagger P<0.01 ; \quad \S P<0.001$; all $P$ values were compared to normal by Student $t$ test.

(Richmond, IN). The control diet contained vitamin-free casein (21\%), sucrose (15\%), non-nutritive fiber (3\%), corn oil (5\%), lard (5\%), dextrin (43.65\%), DL-methionine $(0.15 \%)$, vitamin mixture $(2 \%)$, choline chloride $(0.20 \%)$, and mineral mixture (5\%). A fat-free diet was obtained by omitting the lipid entirely and using extracted casein. The caloric value of the lipid component was met by increasing the content of sucrose to $68.6 \%$ and eliminating the dextrin. Before use, these diets were analyzed for fatty acid content using the methods outlined below. The control diet contained $20 \mathrm{mg}$ linoleate $/ \mathrm{g}$ food and $0.1 \mathrm{mg}$ arachidonate $/ \mathrm{g}$ food. The fat-free diet contained $9 \mu \mathrm{g}$ linoleate/g food and no detectable arachidonate.

Dietary manipulations. 19-d-old weanling male Sprague-Dawley rats were placed on the fat-free diet for 14-24 wk and control rats were placed on the control rat chow. The two dietary groups were housed separately and allowed ad libitum access to water. Two separate batches of fat-deprived animals were studied, one for determination of volatile anesthetic potency and lipid analysis, and the second for fatty acid supplementation studies and subsequent determination of anesthetic potency and lipid composition. Fatty acid supplementation was begun $24 \mathrm{wk}$ after initiation of the fat-free diet. Animals were first supplemented with linolenate $(18: 3 \omega 3)$ by administering $200 \mathrm{mg}$ of methyl linolenate intraperitoneally twice daily for a total of 15 doses (9). Halothane potency was determined in ten of these animals and three of the animals were sacrificed for lipid analysis. The remaining ten animals were subsequently supplemented with linoleate (18:2 $\omega 6$ ) by administering $200 \mathrm{mg}$ of ethyl linoleate intraperitoneally twice daily for a total of 19 doses (9). Halothane potency was measured in these animals, and three were sacrificed for lipid analysis.

Determination of anesthetic potency. To measure anesthetic potency, rats were placed in a 0.2 liter chamber which covered the animals' heads. This chamber was equipped with a rubber gasket which formed a seal around the animal's shoulders. Anesthetic gases (in 100\% oxygen) were delivered to the chamber either from a copper kettle vaporizer (halothane, isoflurane, and methoxyflurane) or from calibrated rotameter flow meters (cyclopropane). Inspired concentrations of halothane, isoflurane, and methoxyflurane were measured by flame ionization detection (10). Gas concentrations were measured both in the delivery tubing and in the anesthetizing chambers, and were in all cases the same. Inspired concentrations of cyclopropane were based solely on rotameter settings. Anesthesia was induced for 20 min with $0.5 \%$ (vol \%) methoxyflurane,

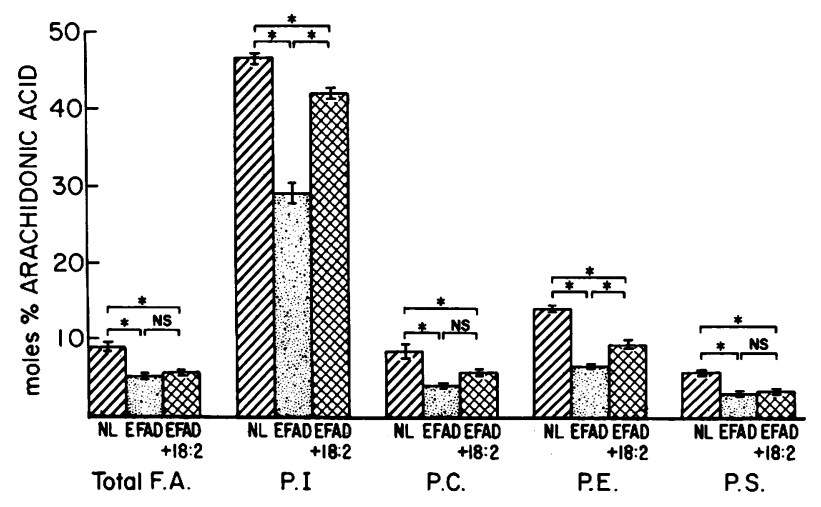

Figure 1. Distribution of arachidonic acid in brain phosphoglycerides following linoleic acid supplementation of fat-deprived rats. Fatty acid methyl esters were generated from either a lipid extract of whole rat brain or from individual phosphoglycerides separated from the lipid extract (as described in Table II). Fatty acid methyl esters were identified and quantitated by gas chromatography and flame ionization detection. Brains were analyzed from rats on a normal diet (NL), a fatfree diet (EFAD), or a fat-free diet for 24 wk followed by short-term parenteral supplementation with linoleic acid (EFAD + 18:2). Results are expressed as mol \% of arachidonate (as percent of total fatty acid [F.A.] methyl esters) \pm SEM ( $n=3$ for all determinations). Data were analyzed by a one-way analysis of variance and Tukey's multiple comparison test on the means. ${ }^{*} P<0.05$. 


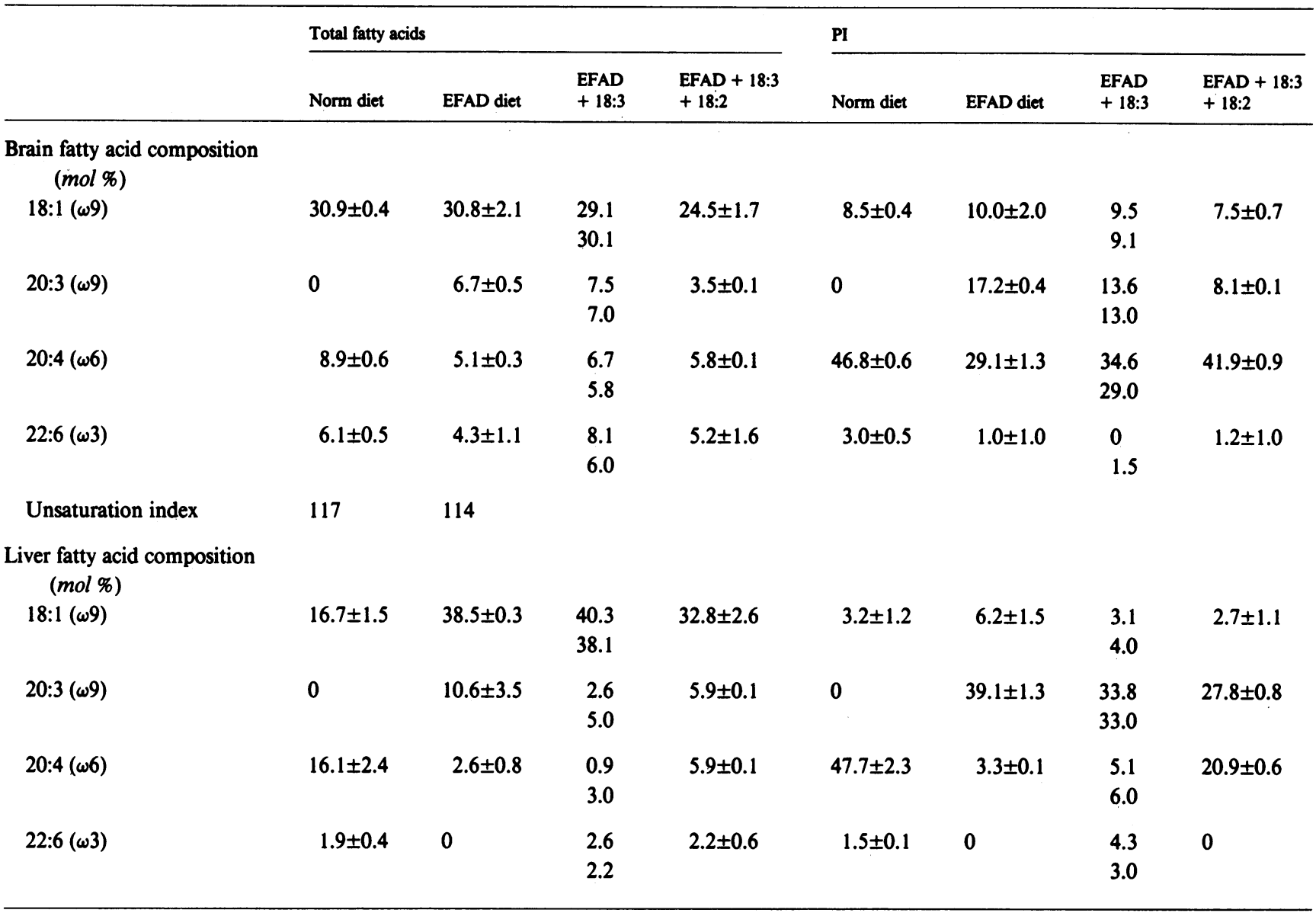

Fatty acid composition of brain and liver phosphoglycerides in EFAD rats, age-matched rats on a normal diet (NORM), EFAD rats supplemented with linolenic acid (EFAD + 18:3), and EFAD rats sequentially supplemented with linolenic and then linoleic acid (EFAD + 18:3 + 18:2). Lipids were extracted from the livers and brains of rats on a normal diet or a fat-free diet. Fatty acid methyl esters were generated from the lipid extract and identified and quantitated by gas chromatography. PI, PC, PE, and PS were separated from the lipid extracts by two-dimensional thin-layer chromatography. Fatty acid methyl esters were generated from each of the phosphoglycerides and analyzed by gas chromatography. All fatty acids that were $>1 \%$ of total fatty acids were analyzed; only fatty acids the content of which was significantly altered by diet are presented in this table. Results are expresssed as mol \% of total fatty acid methyl esters \pm SEM $(n=3$ for all determinations, except EFAD $+18: 3$ where $n=2)$.

$3 \%$ halothane, $3 \%$ isoflurane, or $30 \%$ cyclopropane. During induction, a hemostat was briefly applied to the middle of the rat tails every $10 \mathrm{~s}$ to determine the time to loss of tail-flick response. Following induction, the concentration of the anesthetics was reduced every $20 \mathrm{~min}$ by stepwise decrements until the rats responded with movement to the 30-s application of a hemostat to the base of the tail (11). The decrements of concentration used were $\sim 10 \%$ of the $\mathrm{ED}_{50}{ }^{1}$ value (vol \%) for each of the gases in normal rats. To ensure the validity of these determinations, rats that responded to tail clamp were reanesthetized for $20 \mathrm{~min}$ at the lowest concentration that had prevented tail clamp response, and subsequently, at the highest anesthetic concentration that had permitted tail clamp response. Response to noxious stimulus were verified at each of these concentrations. ED 5 was defined as the mean of the lowest inspired concentration of anesthetic preventing and the highest concentration permitting movement in response to tail clamp (11).

Fatty acid analysis. Liver and brains were removed from rats on a

1. Abbreviations used in this paper: $\mathrm{ED}_{\mathbf{5}}$, mean of the lowest inspired concentration of anesthetic preventing and the highest concentration permitting movement in response to tail clamp; EFAD, essential fatty acid-deficient; PC, phosphatidylcholine; PE, phosphatidylethanolamine; PI, phosphatidylinositol; PS, phosphatidylserine. normal diet, a fat-free diet, or a fat-free diet followed by parenteral fatty acid supplementation. The organs were weighed and lipids were extracted by the method of Bligh and Dyer (12) and resuspended at a uniform concentration in chloroform:methanol, 2:1 (vol/vol). For total fatty acid analysis, an internal standard (21:0 fatty acid) was added to the lipid from $10 \mathrm{mg}$ of tissue, and fatty acid methyl esters were prepared by heating the lipid extract in $14 \% \mathrm{BF}_{3}$ in methanol with benzene added as a solvent. Methyl esters were extracted into hexane and isolated by thin layer chromatography with a solvent system consisting of hexane/diethyl ether/acetic acid (75:5:1). Fatty acid methyl esters were located by comigration with methyl palmitoleate and eluted from the silica gel with hexane. Methyl esters were separated and quantitated by isothermal gas chromatography $\left(200^{\circ} \mathrm{C}\right)$ using a Varian 3700 gas chromatograph containing a glass column $(6 \mathrm{ft} \times 4 \mathrm{~mm})$ packed with $10 \%$ SP-2340 on 100: 120 Chromosorb (Supelco, Inc.) and a $\mathrm{N}_{2}$ flow rate of $40 \mathrm{ml} / \mathrm{min}$. Methyl esters were detected by flame ionization $\left(280^{\circ} \mathrm{C}\right)$ and identified by comigration with authentic standards. Fatty acid methyl ester retention times and quantitation were computed by a Varian 4270 integrator interfaced with the gas chromatograph.

For phospholipid fatty acid analysis, the lipid from $5 \mathrm{mg}$ of tissue was separated into the component phospholipids by the method of Kennerly et al. (13). Briefly, the lipid extract was applied to the corner of a high-performance silica gel plate and separated by two-dimensional thin- 
Table II. (Continued)

\begin{tabular}{|c|c|c|c|c|c|c|c|c|c|c|c|}
\hline \multicolumn{4}{|l|}{ PC } & \multicolumn{4}{|l|}{ PE } & \multicolumn{4}{|l|}{ PS } \\
\hline Norm diet & EFAD diet & $\begin{array}{l}\text { EFAD } \\
+18: 3\end{array}$ & $\begin{array}{l}\text { EFAD + 18:3 } \\
+18: 2\end{array}$ & Norm diet & EFAD diet & $\begin{array}{l}\text { EFAD } \\
+18: 3\end{array}$ & $\begin{array}{l}\text { EFAD + 18:3 } \\
+18: 2\end{array}$ & Norm diet & EFAD diet & $\begin{array}{l}\text { EFAD } \\
+18: 3\end{array}$ & $\begin{array}{l}\text { EFAD + 18:3 } \\
+18: 2\end{array}$ \\
\hline $32.9 \pm 0.5$ & $37.3 \pm 0.3$ & $\begin{array}{l}35.9 \\
35.1\end{array}$ & $35.3 \pm 0.7$ & $18.5 \pm 0.5$ & $20.8 \pm 0.6$ & $\begin{array}{l}15.2 \\
18.0\end{array}$ & $17.6 \pm 1.4$ & $20.8 \pm 0.1$ & $21.5 \pm 1.6$ & $\begin{array}{l}16.0 \\
18.6\end{array}$ & $18.9 \pm 1.3$ \\
\hline 0 & $4.2 \pm 0.3$ & $\begin{array}{l}3.7 \\
3.9\end{array}$ & $\pm 2.1 \pm 0.4$ & 0 & $8.8 \pm 0.4$ & $\begin{array}{r}10.1 \\
9.0\end{array}$ & $6.8 \pm 0.7$ & 0 & $5.7 \pm 0.3$ & $\begin{array}{l}5.1 \\
5.6\end{array}$ & $6.0 \pm 0.4$ \\
\hline $8.5 \pm 1.0$ & $4.2 \pm 0.1$ & $\begin{array}{l}4.5 \\
4.2\end{array}$ & $5.8 \pm 0.3$ & $14.3 \pm 0.3$ & $6.7 \pm 0.1$ & $\begin{array}{l}8.1 \\
7.6\end{array}$ & $9.6 \pm 0.5$ & $5.8 \pm 0.2$ & $2.9 \pm 0.2$ & $\begin{array}{l}2.4 \\
3.0\end{array}$ & $3.3 \pm 0.1$ \\
\hline $5.4 \pm 0.5$ & $1.4 \pm 0.6$ & $\begin{array}{l}3.7 \\
4.0\end{array}$ & $4.8 \pm 0.6$ & $24.6 \pm 0.9$ & $11.4 \pm 1.0$ & $\begin{array}{l}18.7 \\
20.5\end{array}$ & $22.7 \pm 1.7$ & $24.3 \pm 1.5$ & $15.7 \pm 1.0$ & $\begin{array}{l}23.0 \\
21.4\end{array}$ & $21.0 \pm 1.7$ \\
\hline $6.7 \pm 0.7$ & $29.9 \pm 1.4$ & $\begin{array}{l}20.1 \\
18.0\end{array}$ & $13.9 \pm 1.2$ & $6.8 \pm 0.8$ & $19.2 \pm 0.7$ & $\begin{array}{l}7.7 \\
8.1\end{array}$ & $6.2 \pm 0.8$ & $4.9 \pm 2.8$ & $9.0 \pm 2.0$ & $\begin{array}{l}4.6 \\
5.1\end{array}$ & $4.0 \pm 0.4$ \\
\hline 0 & $21.8 \pm 0.6$ & $\begin{array}{r}8.2 \\
12.0\end{array}$ & $11.3 \pm 0.6$ & 0 & $27.6 \pm 1.8$ & $\begin{array}{l}5.6 \\
7.0\end{array}$ & $6.9 \pm 0.5$ & 0 & $19.2 \pm 1.5$ & $\begin{array}{l}3.4 \\
5.0\end{array}$ & $4.3 \pm 0.8$ \\
\hline $32.3 \pm 3.3$ & $3.4 \pm 0.5$ & $\begin{array}{l}2.2 \\
4.1\end{array}$ & $14.0 \pm 0.6$ & $35.6 \pm 2.4$ & $12.6 \pm 0.3$ & $\begin{array}{l}5.2 \\
8.0\end{array}$ & $29.1 \pm 1.3$ & $40.0 \pm 4.7$ & $22.0 \pm 2.2$ & $\begin{array}{r}6.3 \\
11.0\end{array}$ & $26.4 \pm 0.1$ \\
\hline $4.6 \pm 0.4$ & $0.6 \pm 0.1$ & $\begin{array}{l}7.9 \\
9.0\end{array}$ & $8.7 \pm 1.5$ & $9.0 \pm 0.8$ & $2.8 \pm 0.5$ & $\begin{array}{l}19.2 \\
18.0\end{array}$ & $18.2 \pm 1.9$ & $9.0 \pm 1.2$ & $2.4 \pm 1.2$ & $\begin{array}{l}27.4 \\
21.0\end{array}$ & $17.0 \pm 2.1$ \\
\hline
\end{tabular}

layer chromatography. Solvent system 1 consisted of chloroform/methanol/ammonium hydroxide (58\%)/water, 65:35:5:0.6 (vol/vol) and system 2 consisted of chloroform/acetone/methanol/acetic acid/water; 45:19: 10:15:4 (vol/vol). Phospholipid spots were visualized with rhodamine $G$ $(0.012 \%)$ and an internal standard (21:0 methyl ester) was added to each spot. The spots were individually scraped from the plates and eluted from the silica gel with chloroform. Fatty acid methyl esters were prepared by reacting the phospholipids for 10 min with $0.5 \mathrm{~N} \mathrm{NaOH}$ in methanol and then quenching with $6 \mathrm{~N} \mathrm{HCl}$. Fatty acid methyl esters were extracted into chloroform and analyzed by gas chromatography as described above. Phospholipids were quantitated by measurement of inorganic phosphate (14).

\section{Results}

Rats on a fat-free diet exhibited no behavioral abnormalities; they were alert, active, and free of any gross neurologic deficits. The fat-deprived animals did show the characteristic signs of essential fatty acid deficiency, namely a scaling dermatitis, partial hair loss, and growth retardation (8). Rats on the fat-free diet weighed $306 \pm 18 \mathrm{~g}$ (mean \pm SEM), whereas control rats on a normal diet weighed $425 \pm 14 \mathrm{~g}$.
Essential fatty acid-deficient (EFAD) rats were significantly more sensitive to all of the volatile anesthetics tested than rats on a normal diet (Table I $A$ ). These differences in anesthetic sensitivity were not the result of differences in weight, since halothane sensitivity was tested in a second batch of EFAD animals where weight-matched rats on a normal diet were used as controls (Table I $B$ ). The halothane $\mathrm{ED}_{50}$ values of age-matched rats on a normal diet (1.24 $\pm 0.04 \mathrm{vol} \%)$ and weight-matched rats on a normal diet $(1.21 \pm 0.02)$ were not significantly different.

The anesthetics used in this study were selected to span a wide range of lipid solubility and anesthetic potency. There was a significant correlation between the lipid solubility (oil/gas partition coefficient) of the agents tested and the percent change in anesthetic sensitivity caused by fat deprivation $(r=0.97, P$ $<0.05$ ). The time to loss of tail-flick response (induction time) did not differ between normal and EFAD rats for any anesthetic tested (Table I), which suggests that the rate of anesthetic uptake into blood and subsequently into brain was not affected by EFAD.

To determine if the alterations in anesthetic potency observed in EFAD animals were due to the absence of a specific fatty 
acid, fatty acid supplementation studies were performed. In these studies, halothane was used as an index anesthetic. Following supplementation of EFAD animals with linolenic acid (18:3 $\omega 3)$, no change in halothane sensitivity was observed (Table I $B$ ). Thus animals deficient only in $\omega 6$ fatty acids showed the same increased sensitivity to halothane as EFAD animals. Supplementation of these rats with linoleic acid $(18: 2 \omega 6)$ resulted in a dramatic alteration in halothane sensitivity (Table I $B$ ). Linoleic acid-supplemented EFAD rats were significantly less sensitive to halothane than were control rats.

Lipid analyses. Brains from normal and EFAD rats were removed and analyzed for total fatty acid composition and the fatty acid composition of phosphatidylinositol (PI), phosphatidylcholine (PC), phosphatidylethanolamine (PE), and phosphatidylserine (PS). Livers from these animals were also analyzed to validate essential fatty acid deficiency. Fatty acid analysis (Table II) showed that arachidonic acid $(20: 4 \omega 6)$ was the major $\omega 6$ fatty acid in brain and liver. Brain PI had the highest molar percentage of arachidonate of the brain phosphoglycerides, whereas hepatic arachidonate was evenly distributed in all phos-

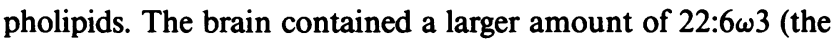
major membrane $\omega 3$ fatty acid) than did liver, and in both organs, this fatty acid was preferentially distributed in PE and PS. EFAD

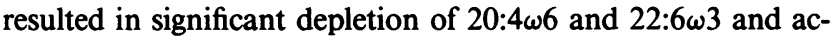
cumulation of $\omega 9$ series polyunsaturated fatty acids $(18: 1,20: 3)$ in all phospholipids in both organs. Quantitative analysis (data not shown) indicated that the fatty acid content (micrograms per milligram tissue) paralleled the fatty acid composition (mol $\%$ ) for all of the lipid classes studied. Interestingly, while brain fatty acid composition was markedly altered by EFAD, brain unsaturation indices (sum of mol $\%$ of each fatty acid $\times$ number of double bonds in each fatty acid) did not differ in normal and EFAD rats. It should be noted that unsaturation indices were calculated from all of the fatty acids in the lipid extract, not just those shown in Table II.

Brains and livers from EFAD animals following fatty acid supplementation were also analyzed for fatty acid composition. These fatty acid analyses showed that supplementation with either linolenate or linoleate resulted in accumulation of chain elongation/desaturation products of the administered fatty acids, but minimal accumulation of the administered compounds. Supplementation of EFAD rats with linolenate (Table II) caused: (a) complete repletion of 22:6 33 in brain distributing primarily in PE and PS; (b) supranormal levels of hepatic 22:6w3 in PC, PE, and PS; (c) no substantive changes in $20: 4 \omega 6$ or $20: 3 \omega 9$ in brain; and $(d)$ a decrease in the molar percentage of hepatic 20: $3 \omega 9$. Thus, while the linolenate supplementation protocol used completely normalized the $\omega 3$ fatty acid content of the EFAD rat brain, it did not normalize halothane sensitivity. These results indicate that brain $\omega 3$ fatty acid deficiency is not responsible for EFAD-induced alterations in anesthetic potency.

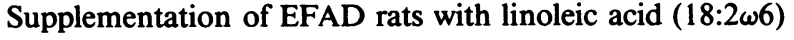
resulted in partial repletion of arachidonate $(20: 4 \omega 6)$ and partial depletion of 20:3 $\omega 9$ in both liver and brain. In liver (Table II), reconstitution of arachidonate and depletion of 20:3 $\omega 9$ occurred nonspecifically in all phospholipids. In brain, linoleate supplementation resulted in only a small increase in arachidonate as a percentage of total fatty acids. Interestingly, the small amount of arachidonate that was incorporated into brain lipids was nonuniformly distributed in the various phosphoglycerides (Fig. 1). Linoleate supplementation resulted in a preferential repletion of arachidonate in brain PI, a partial repletion of arachidonate in PE, and no significant repletion of PC or PS. Linoleic acid

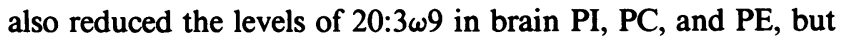
not PS.

\section{Discussion}

The dietary manipulations used in this study appear to have specifically altered brain fatty acid composition, independent of other alterations in brain lipid structure. Previous studies using stringently fat-restricted diets showed no significant alteration in the quantity of individual phosphoglycerides, cholesterol, gangliosides, protein, or DNA in rat brain (16-17). These same studies show changes in brain fatty acid composition similar to those reported here (17-18). We have also quantitated the brain content of PI, PC, PE, PS, and sphingomyelin in normal and EFAD rat brain and found that our diet does not affect the content of any of these lipids (data not shown). Additionally, the present results are in agreement with the previously reported finding that EFAD does not alter brain unsaturation index (19). Thus, the effects of fat restriction on anesthetic action are likely due to altered brain fatty acid composition, and not to changes in brain membrane unsaturation or phosphoglyceride content.

While our results clearly indicate that specific alterations in the fatty acid composition of the brain alters anesthetic potency, further clarification of the mechanistic role of fatty acids in anesthetic mechanism can only be inferred from the present data. The correlation between the magnitude of EFAD-induced alterations in anesthetic potency and the oil/gas partition coefficients of the anesthetics tested suggests the possibility that EFAD may alter the solubility of anesthetics in neuronal membranes. The specific reversal of increased halothane sensitivity following the incorporation of small amounts of $\omega 6$ fatty acids argues against such a bulk effect. Nonetheless, direct measurements of anesthetic solubility in normal and EFAD brain will be necessary to resolve this issue.

The finding that linoleate reversal of increased halothane sensitivity was associated with preferential incorporation of arachidonate into brain phosphatidylinositol suggests an alternative mechanistic interpretation of the data. PI degradation (turnover) is an important transducing system in the cellular response to a variety of agonists (20), and several neurotransmitters have been shown to stimulate brain PI turnover (21-23). Furthermore, rat brain is exceedingly rich in PI catabolizing enzymes $(24,25)$. If indeed the action of some neurotransmitters is mediated through PI turnover, this metabolic pathway would be a logical target for central nervous system depressants, such as the volatile anesthetics. Arachidonyl PI could be important either because it serves as a substrate for a membrane enzyme directly inhibited by anesthetics, or because there is a direct interaction between arachidonyl PI and the volatile anesthetics.

In this regard it is important to note that while linoleate supplementation caused a rebound decrease in halothane sensitivity (Table I B), the molar percentage of arachidonate in PI (or other phospholipids) did not increase beyond control (Fig. 1). The fatty acid analyses in this study were performed on whole rat brain and, as such, contained large amounts of non-neuronal lipid, including myelin. The fatty acids of myelin are thought to have a slower rate of turnover than neuronal fatty acids (26, 27). Thus, while the data do not specifically describe the effects of dietary manipulation on neuronal fatty acid composition, the 
compositional changes in neurons following short-term fatty acid supplementation may be more extreme than the changes seen in whole brain. Indeed, the work of Galli et al. (28) showed that realimentation of fat-deprived rats resulted in a rebound increase in $\omega 6$ fatty acids in the synaptosomal, but not the myelin fraction of brain.

While the results of our study stress the importance of lipid structure in anesthetic mechanism, it should not be inferred from these data that anesthetics do not interact with target membrane proteins. The activity of many integral membrane proteins is modulated by the lipid composition of the membrane in which the protein resides (29). The milieu in which a potential target protein resides could be changed by specific fatty acid compositional alterations, which influence the interaction between anesthetic and protein.

In summary, our data demonstrate that brain essential fatty acid deficiency potentiates volatile anesthetic action in rats, and that this potentiation is specifically reversed by linoleic acid supplementation. These data describe, for the first time, a model in which changes in membrane structure can be correlated with changes in in vivo anesthetic effect and provide the first direct evidence that the precise lipid composition of the brain can alter anesthetic effect. Further study of this model should lead to increased understanding of the mechanism of volatile anesthetic action.

\section{References}

1. Seeman, P. 1972. The membrane actions of anesthetics and tranquilizers. Pharmacol. Rev. 24:583-655.

2. Janoff, A. S., M. J. Pringle, and K. W. Miller. 1981. Correlation of general anesthetic potency with solubility in membranes. Biochim. Biophys. Acta. 649:125-128.

3. Miller, K. W., and K. Y. Pang. 1976. General anesthetics can selectively perturb lipid bilayer membranes. Nature (Lond.). 263:253255.

4. Trudell, J. R. 1977. A unitary theory of anesthesia based on lateral phase separations in nerve membrane. Anesthesiology. 46:5-10.

5. Richards, C. D., K. Martin, S. Gregory, C. A. Keightly, T. R. Hesketh, G. A. Smith, C. B. Warren, and J. C. Metcalfe. 1978. Degenerate perturbations of protein structure as the mechanism of anaesthetic action. Nature (Lond.). 276:775-779.

6. Frank, N. P., and W. R. Lieb. 1982. Molecular mechanisms of general anesthesia. Nature (Lond.). 300:487-493.

7. Trudell, J. R. 1985. Is there light at the end of the tunnel? Anesth. Analg. 64:385.

8. Holman, R. T. 1969. Essential fatty acid deficiency. Prog. Chem. Fats Other Lipids. 9:275-348.

9. Rahm, J. J., and R. T. Holman. 1964. Studies of the metabolism of polyunsaturated acids by short-term experiments. J. Nutrition. 84: 149-152.
10. Lowe, H. J. 1964. Flame ionization detection of volatile organic anesthetics in blood, gases and tissues. Anesthesiology. 25:808-814.

11. Munson, E. S., J. C. Hoffman, and C. A. Difazio. 1968. The effect of hypothyroidism and hyperthyroidism on cyclopropane requirement in rats. Anesthesiology. 29:1094-1098.

12. Bligh, E. G., and W. J. Dyer. 1959. A rapid method for total lipid extraction and purification. Can. J. Biochem. Physiol. 37:911-917.

13. Kennerly, P. A., T. J. Sullivan, and C. W. Parker. 1979. Activation of phospholipid metabolism during mediator release from stimulated rat mast cells. J. Immunol. 122:152-159.

14. Vaskovsky, V. E., E. Y. Kostetsky, and I. M. Vasendin. 1975. A universal reagent for phospholipid analysis. J. Chromatogr. 114:129141.

15. Dripps, R. D., J. E. Eckenhoff, and L. D. Vandam. 1977. Introduction to Anesthesia. Fifth ed. W. B. Saunders Co., Philadelphia. 118.

16. Galli, C., H. B. White, and R. Paoletti. 1970. Brain lipid modifications induced by essential fatty acid deficiency in growing male and female rats. J. Neurochem. 17:347-355.

17. Odutaga, A. A. 1977. Recovery of brain from deficiency of essential fatty acids in rats. Biochim. Biophys. Acta. 487:1-9.

18. Odutaga, A. A. 1981. Reversal of brain essential fatty-acid deficiency in the rat by dietary linoleate, linolenate and arachidonate. Int. J. Biochem. 13:1035-1038.

19. Trapp, B. D., and J. Bernsohm. 1977. Changes in phosphoglyceride fatty acids of rat brain induced by linoleic and linolenic acids after pre- and post-natal fat deprivation. J. Neurochem. 28:1009-1013.

20. Berridge, M. J. 1984. Inositol trisphosphate and diacylglycerol as second messengers. Biochem. J. 220:345-360.

21. Griffin, H. D., J. N. Hawthorne, and M. Sykes. 1979. A calcium requirement for the phosphatidylinositol response following activation of presynaptic muscarinic receptors. Biochem. Pharmacol. 28:1143-1147.

22. Berridge, M. J., R. M. C. Dawson, C. P. Downes, J. P. Heslop, and R. F. Irvine. 1983. Changes in the levels of inositol phosphates after agonist-dependent hydrolysis of membrane phosphoinositides. Biochem. J. 212:473-482.

23. Abdel-Latiff, A. A., R. A. Akhtar, and J. P. Smith. 1978. Cyclitols and Phosphoinositides. W. W. Wells and F. Eisenberg, editors. Academic Press, Inc., New York. 121-143.

24. Irvine, R. F., N. Hemington, and R. M. C. Dawson. 1979. The calcium-dependent phosphatidylinositol-phosphodiesterase of rat brain. Eur. J. Biochem. 99:525-530.

25. Kikkawa, U., Y. Takai, R. Minakuchi, S. Inohara, and Y. Nishizuka. 1982. Calcium-activated, phospholipid-dependent protein kinase from rat brain. J. Biol. Chem. 257:13341-13348.

26. Sun, G. Y. 1973. The turnover of phosphoglycerides in the subcellular fractions of mouse brain: a study using $1-{ }^{14} \mathrm{C}$ oleic acid as a precursor. J. Neurochem. 21:1083-1092.

27. Miller, S. L., and P. Morell. 1978. Turnover of phosphatidylcholine in microsomes and myelin in brains of young and adult rats. $J$. Neurochem. 31:771-777.

28. Galli, C., H. B. White, and R. Paoletti. 1971. Lipid alterations and their reversion in the central nervous system of growing rats deficient in essential fatty acids. Lipids. 6:378-387.

29. Sandermann, H. 1978. Regulation of membrane enzymes by lipids. Biochim. Biophys. Acta. 515:209-237. 